Estimating detection rates for the LIGO-Virgo search for gravitational-wave burst counterparts to gamma-ray bursts using inferred local GRB rates

This article has been downloaded from IOPscience. Please scroll down to see the full text article.

2009 Class. Quantum Grav. 26204017

(http://iopscience.iop.org/0264-9381/26/20/204017)

View the table of contents for this issue, or go to the journal homepage for more

Download details:

IP Address: 131.251.133.25

The article was downloaded on 04/04/2012 at 12:52

Please note that terms and conditions apply. 


\title{
Estimating detection rates for the LIGO-Virgo search for gravitational-wave burst counterparts to gamma-ray bursts using inferred local GRB rates
}

\author{
I Leonor ${ }^{1}$, P J Sutton ${ }^{2}$, R Frey ${ }^{1}$, G Jones ${ }^{2}$, S Márka $^{3}$ and Z Márka ${ }^{3}$ \\ ${ }^{1}$ University of Oregon, Eugene, OR 97403, USA \\ 2 Cardiff University, Cardiff, CF24 3AA, UK \\ ${ }^{3}$ Columbia University, New York, NY 10027, USA \\ E-mail: ileonor@uoregon.edu
}

Received 1 June 2009

Published 6 October 2009

Online at stacks.iop.org/CQG/26/204017

\begin{abstract}
One of the ongoing searches performed using the LIGO-Virgo network of gravitational-wave interferometers is the search for gravitational-wave burst (GWB) counterparts to gamma-ray bursts (GRBs). This type of analysis makes use of GRB time and position information from gamma-ray satellite detectors to trigger the GWB search, and the GWB detection rates possible for such an analysis thus strongly depend on the GRB detection efficiencies of the satellite detectors. Using local GRB rate densities inferred from observations which are found in the science literature, we calculate estimates of the GWB detection rates for different configurations of the LIGO-Virgo network for this type of analysis.
\end{abstract}

PACS numbers: $\quad$ 04.30.Tv, 04.80.Nn, 95.40.Sf, 95.85.Sz, 98.70.Rz, 95.85.Pw

\section{Introduction}

Gravitational-wave bursts (GWBs) associated with gamma-ray bursts (GRBs) are among the primary types of signals targetted by the LIGO-Virgo network of gravitational-wave (GW) interferometers. Searches for GWB counterparts to GRBs have been performed for the second LIGO science run up to the fifth LIGO and first Virgo science runs (which will be referred to jointly in this paper as the S5/VSR1 run) [1-3]. The method employed by LIGO-Virgo to search for GWB counterparts to GRBs relies on time and position information from gamma-ray satellite detectors, i.e. GRB detections by gamma-ray satellite experiments trigger corresponding LIGO-Virgo GWB counterpart searches. The method thus strongly depends on the detection efficiencies of the gamma-ray detectors which are in operation contemporaneously with a particular LIGO-Virgo science run. 
For example, during the LIGO-Virgo S5/VSR1 2-year run, in which the LIGO interferometers were operating at their initial design sensitivities, the Swift satellite's BAT detector [4, 5] was detecting GRBs at a rate of about 100 per year. The BAT detector's field of view of $1.4 \mathrm{sr}$ at $>50 \%$ coding (or $2.2 \mathrm{sr}$ at $>10 \%$ coding) results in a detection rate which is smaller than that which was possible with the BATSE detector on board the CGRO satellite [6]. For future science runs of the LIGO-Virgo network, it will be desirable that GRBs be detected by gamma-ray satellite experiments with high efficiency in order to maximize GWB detection probabilities using such a triggered search. During the LIGO-Virgo S6/VSR2 run, in which the interferometers will be operating at higher sensitivity compared to their initial configuration during the S5/VSR1 run, most of the GRB triggers will be coming from the GBM detector on board the Fermi satellite [7, 8]. Together with the GRB triggers from BAT, this will result in a factor of $\approx 3$ increase in the rate of on-the-fly GRB triggers compared with the GRB trigger rate during the S5/VSR1 run.

In this paper, we estimate the possible detection rates for GWB counterparts to GRBs for different configurations of the LIGO-Virgo network using a triggered search, and using local GRB rates inferred from GRB observations which are found in the science literature.

\section{Local GRB rate densities}

Several references have independently inferred local GRB rates from observations. The references considered here generally base their analysis on the $V / V_{\max }$ method [9-12], in which the peak flux distribution of GRBs is modeled as the convolution of the local comoving GRB luminosity function and the comoving GRB production rate, both of which are unknown functions. Because of BATSE's all-sky field of view, the large sample collected during its mission, and the completeness of this sample (i.e. it is assumed that all GRBs above a threshold flux were detected), its GRB sample is usually used to form the observed GRB peak flux distribution to which the parameters of the luminosity function are fitted. The normalization of the rate function, which is the local GRB rate density, $\rho_{0}$, can be determined by setting the integrated peak flux distribution equal to the effective number of GRBs per year in the BATSE sample that was used.

The model used for the GRB formation rate, $R_{\mathrm{GRB}}(z)$, generally depends on the GRB class being considered, i.e. long- or short-duration GRBs. For long-duration GRBs, whose progenitors are thought to be core-collapse supernovae, $R_{\mathrm{GRB}}(z)$ is assumed to follow the star-formation rate of the Universe, for which standard models are found in the literature. For short-duration GRBs, which are thought to be due to compact binary mergers, i.e. double neutron star (NS-NS) or neutron star-black hole (NS-BH) mergers, the model for $R_{\mathrm{GRB}}(z)$ would typically track the star-formation rate with a distribution of delay times to account for the time it takes for a binary system to form, inspiral—due to emission of GW radiation — and eventually merge.

Additionally, for short GRBs, secure redshift measurements are available for a small sample and provide evidence that the redshift distribution of short GRBs peaks at a lower redshift compared to long GRBs. These redshift measurements are used to further constrain the fits to the rate models and, as a result, local rate densities obtained with this constraint are higher than those from previous calculations [13].

Moreover, there is also limited evidence for a separate population of low-luminosity, long-duration GRBs which are inferred to be at closer distances than high-luminosity GRBs, and which cannot be accounted for by a single-component luminosity function [15]. Correspondingly, the inferred local rate density for this putative population of GRBs is also higher than that for high-luminosity, long-duration GRBs. 
Table 1. Local GRB rate densities (isotropic-equivalent) inferred from observations.

\begin{tabular}{|c|c|c|c|c|}
\hline \multirow[b]{2}{*}{ Reference } & \multirow[t]{2}{*}{$\rho_{0}$} & \multirow{2}{*}{$\begin{array}{l}\text { Uncertainty } \\
\left(\mathrm{Gpc}^{-3} \mathrm{yr}^{-1}\right)\end{array}$} & \multirow{2}{*}{$\begin{array}{l}\text { Effective full-sky } \\
\text { number of GRBs } \\
\left(\mathrm{yr}^{-1}\right)\end{array}$} & \multirow[b]{2}{*}{ GRB class ${ }^{b}$} \\
\hline & & & & \\
\hline [9] & $0.5^{\mathrm{c}}$ & - & 694 & Long and short ${ }^{\mathrm{d}}$ \\
\hline [14] & 0.44 & $\sim 0.3^{\mathrm{e}}$ & $\approx 700$ & Long \\
\hline [15] & $\sim 200$ & $\sim 100$ & - & Long, low-luminosity \\
\hline [13] & $\sim 8-30$ & $(-4,+40),(-20,+50)^{\mathrm{f}}$ & $\approx 108$ & Short \\
\hline [16] & $\sim 3^{g}$ & $\sim 1$ & $\approx 108$ & Short \\
\hline
\end{tabular}

${ }^{a}$ Calculated using the total number of GRBs in the sample, the effective observation time and the effective sky coverage.

${ }^{\mathrm{b}}$ The classification into short- and long-duration GRBs follows the usual convention, i.e. $T_{90}<2 \mathrm{~s}$ and $T_{90}>2 \mathrm{~s}$ for short and long GRBs, respectively, where $T_{90}$ is the time during which $90 \%$ of the counts are detected.

c [9] does not explicitly quote uncertainties for the inferred local GRB rates, but the rates are $0.48,0.51$ and $0.72 \mathrm{Gpc}^{-3} \mathrm{yr}^{-1}$ for three different models of star formation. The value $0.5 \mathrm{Gpc}^{-3} \mathrm{yr}^{-1}$ is often quoted in the literature.

${ }^{\mathrm{d}}$ Since the GRB sample was from BATSE DISCLA data on a timescale of $1024 \mathrm{~ms}$, the GRB durations are larger than $1 \mathrm{~s}$.

${ }^{\mathrm{e}}$ The inferred local GRB rates in [14] range from $\sim 0.1$ to $0.44 \mathrm{Gpc}^{-3} \mathrm{yr}^{-1}$ depending on the model for star formation, but the value which produces a predicted distribution of redshifts which better matches the observed values is $0.44 \mathrm{Gpc}^{-3} \mathrm{yr}^{-1}$.

${ }^{\mathrm{f}}$ The two sets of $1 \sigma$ uncertainties are for the lower and upper ends of the range, respectively.

${ }^{g}$ [16] calculates the local GRB rate for both primordial and dynamically formed NS-NS and NS-BH binaries. The number quoted in this table is for $\sim 60 \%$ contribution from dynamically formed NS-NS and NS-BH binaries.

We summarize the local rate densities, $\rho_{0}$, from different references in table 1 . Note that these rates are isotropic-equivalent rates inferred from GRB observations, i.e. they are not corrected for the effect of GRB beaming, which would be to increase the inferred local rate density. In estimating the GWB detection rates, it is important to note that there are large uncertainties in the inferred local GRB rates, with an uncertainty of a factor of $\sim 2$ being typical (cf table 1). An advantage of not needing the beaming factor to estimate the GWB detection rates is that no additional uncertainties, due to the inferring values of the beaming factor, are introduced in the estimates.

\section{Estimated GWB detection rates}

We can use the local GRB rate densities to estimate detection rates for GWB counterparts to GRBs using a triggered search and different LIGO-Virgo network configurations. The method generally employed in LIGO-Virgo to search for GWB counterparts to GRBs makes use of the trigger time information provided by a gamma-ray satellite experiment to determine the time interval to analyze using LIGO-Virgo data. The time window used in the search is about a few minutes before and after the GRB trigger time. A fully coherent network search algorithm [19] is used to search for GWB signals. It is also noted that there is a separate method which makes use of binary inspiral templates to search for GW signals from short-duration GRBs [3], and which is not discussed in this paper.

The standard procedure in the triggered search is to set frequency-dependent upper limits on the GW strain amplitude for each GRB analyzed. By making some assumptions on the 
energy emitted in gravitational waves, it is possible to estimate corresponding lower limits on the GRB distances. For isotropic emission of gravitational waves and circularly polarized waves, the luminosity distance is related to the energy, $E_{\mathrm{GW}}$, and the root sum square strain amplitude, $h_{\text {rss }}$, by [2]

$$
D \approx\left(\frac{G}{\pi^{2} c^{3}}\right)^{1 / 2} \frac{E_{\mathrm{GW}}^{1 / 2}}{f_{\mathrm{o}} h_{\mathrm{rss}}},
$$

where $f_{\mathrm{o}}$ is the observed frequency of the waves.

The sensitivity of the fully coherent network search is estimated to be a factor of $\sim 2$ more sensitive than the crosscorrelation-based method that was used in previous searches for GWB counterparts to GRBs [2,3]. The average distance sensitivity of the fully coherent network analysis method used in the triggered search if applied to data taken during the S5/VSR1 run, is thus estimated to be $\sim 15 \mathrm{Mpc}$ at $150 \mathrm{~Hz}$ - the frequency at which the interferometers were most sensitive - and assuming energy emitted in gravitational waves of $0.01 M_{\odot}$. The value of $0.01 M_{\odot}$ was chosen as a reference value because it is a reasonable energy one might expect in the LIGO-Virgo band in various progenitor models [20-27]. The GWB detection rate is then given by

$$
R_{\mathrm{GWB}} \approx \rho_{0}\left(\frac{4}{3} \pi D^{3}\right)
$$

Based on results of past searches, the uncertainty in the estimated average distance sensitivity, $D=15 \mathrm{Mpc}$, for the S5/VSR1 run corresponding to an assumed energy emission of $E_{\mathrm{GW}}=0.01 M_{\odot}$ is estimated to be less than $10 \%$, so that the uncertainty in $R_{\mathrm{GWB}}$ will be dominated by the uncertainty in $\rho_{0}$.

In the case of the S5/VSR1 run, most of the GRB triggers were from Swift. There were 212 GRB triggers during the 2 years in which the S5/VSR1 was ongoing, or $\sim 100 \mathrm{yr}^{-1}$. Of these 212 GRBs, 33 were classified as short GRBs. Studies which compare the peak flux distributions of BATSE and Swift, corrected for the different energy band sensitivities, indicate that the two detectors are sampling the same population of GRBs and have similar sensitivities $[17,18]$. However, the field of view of the Swift BAT detector is smaller than that of BATSE, which accounts for the lower Swift GRB trigger rate. To correct for this difference, the local rate densities given in table 1 have to be divided by a factor given by the ratio of the effective full-sky number of BATSE GRBs per year given in the table to the number of Swift GRBs per year during the S5/VSR 1 run. This factor is $\approx 7$ in the case of both long $(\approx 700 / 90)$ and short $(\approx 108 / 16)$ GRBs.

In the case of the S6/VSR2 run, if the sensitivity goal is achieved, the distance reach of the interferometers will be approximately twice that achieved during the S5/VSR1 run [28] (or a factor of 8 increase in volume reach), it is expected that most of the GRB triggers will be from Fermi, and that there will be a factor of $\approx 3$ increase in the on-the-fly GRB trigger rate compared to the trigger rate during S5/VSR1. This trigger rate is within a factor of 2 of the BATSE rate resulting from on-board triggers and offline analysis, as indicated in table 1. If we assume that additional GRBs will be detected in an offline analysis of Fermi and Swift data, then we can use the GRB rate densities given in table 1 in our calculation of the GWB detection rate estimates. A similar scenario is assumed in the case of Advanced LIGO-Virgo, in which the interferometers are expected to achieve a factor of 10 or more increase in strain amplitude sensitivity over the initial LIGO-Virgo interferometers [28].

For estimating the GWB detection rates, we adopt the local GRB rate densities in $[9,13]$ as central values. A summary of the resulting estimates of the GWB detection rates is given in table 2, where several orders of magnitude improvement in detection rate is evident in the transition from the initial to the advanced configuration of interferometers. To use a different 
Table 2. Estimated detection rates for GWB counterparts to GRBs, calculated using (2), $E_{\mathrm{GW}}=0.01 M_{\odot}$ and $f_{\mathrm{o}}=150 \mathrm{~Hz}$.

\begin{tabular}{llll}
\hline & $\begin{array}{l}\text { Estimated average } \\
\text { distance reach } \\
\text { Detector }\end{array}$ & \multicolumn{2}{c}{ Estimated detection rate $\left(\mathrm{yr}^{-1}\right)$} \\
\cline { 3 - 4 } & $(\mathrm{Mpc})$ & Long GRBs & Short GRBs \\
\hline Initial LIGO-Virgo $^{\mathrm{a}}$ & $\sim 15$ & $\sim 1 \times 10^{-6}$ & $\sim(1.6-6.1) \times 10^{-5}$ \\
Enhanced LIGO-Virgo $^{\mathrm{b}}$ & $\sim 30$ & $\sim 5.7 \times 10^{-5}$ & $\sim(0.9-3.4) \times 10^{-3}$ \\
Advanced LIGO-Virgo $^{\text {Advand }}$ & $\sim 150$ & $\sim 7.1 \times 10^{-3}$ & $\sim 0.11-0.42$ \\
\hline
\end{tabular}

a S5/VSR1 run.

${ }^{\mathrm{b}}$ As an example, we assume that a goal of a factor of $\sim 2$ improvement in strain amplitude sensitivity over the initial LIGO-Virgo interferometers is achieved.

value of the GW energy emission, the scaling goes as

$$
R_{\mathrm{GWB}}=R_{\mathrm{GWB}, \mathrm{ref}}\left(\frac{E_{\mathrm{GW}}}{0.01 M_{\odot}}\right)^{3 / 2}
$$

where $R_{\mathrm{GWB} \text {,ref }}$ are the reference rates given in table 2 .

It is not straightforward at this time to perform the same estimate calculation in the case of a putative population of low-luminosity, long-duration GRBs, because current estimates of the rate density for this population are based on a very small sample, and also because it is not clear at this point if current or future GRB experiments will be detecting nearby lowluminosity GRBs efficiently. In the optimistic scenario that GRB experiments will have the necessary sensitivity to detect these objects, and if we perform the calculation using the local GRB rate densities given in [15], then the corresponding GWB detection rate is $\sim 0.02 \mathrm{yr}^{-1}$ and $\sim 2.8 \mathrm{yr}^{-1}$ for Enhanced LIGO-Virgo and Advanced LIGO-Virgo, respectively. These are certainly more encouraging numbers than those inferred in the case of high-luminosity GRBs, but they should be read critically because of the reasons stated above.

\section{Conclusion}

We have used local GRB rate densities inferred from observations (cf table 1) to estimate the detection rates for GWB counterparts to GRBs using a triggered search and different LIGOVirgo network configurations. These detection rates for $E_{\mathrm{GW}}=0.01 M_{\odot}$ and $f_{\mathrm{o}}=150 \mathrm{~Hz}$ are summarized in table 2. The numbers are encouraging for the detection of GWB counterparts with Advanced LIGO-Virgo. Equation (3) gives the scaling for other assumed energy values. It is again emphasized that the local GRB rate densities have large uncertainties-with an uncertainty factor of $\sim 2$ being typical—and these uncertainties propagate into our estimates of the GWB detection rate through (2). Because of the nature of a triggered search, it was sufficient to use isotropic-equivalent GRB rate densities to calculate our GWB detection estimates. This meant that no additional uncertainties due to inferring beaming factors were introduced in the calculations.

We have also considered local GRB rate densities due to a putative population of nearby, low-luminosity, long-duration GRBs, which give rise to more optimistic estimates of the GWB detection rate. However, due to the very limited sample from which the GRB rate densities were inferred, and the uncertainty in the efficiency with which current and future GRB experiments will be detecting these objects, there is a high uncertainty attached to the estimated GWB detection rate. 


\section{References}

[1] Abbott B et al 2005 Phys. Rev. D 72042002

[2] Abbott B et al 2008 Phys. Rev. D 77062004

[3] Abbott B et al 2008 Astrophys. J. 6811419

[4] Gehrels N et al 2004 Astrophys. J. 611 1005-20

[5] Sakamoto T et al 2008 Astrophys. J. Suppl. 175 179-90

[6] Fishman G J, Meegan C A, Wilson R B, Paciesas W S and Pendleton G N 1992 The Compton Observatory Science Workshop pp 26-34

[7] Stamatikos M, Sakamoto T and Band D L 2008 Nanjing Gamma-Ray Burst Conf. (AIP Conf. Proc. vol 1065) pp 59-62

[8] Band D L 2008 Gamma-Ray Bursts 2007: Proc. Santa Fe Conf. (AIP Conf. Proc. vol 1000) pp 121-124

[9] Schmidt M 2001 Astrophys. J. 552 36-41

[10] Schmidt M 1999 Astrophys. J. 523 L117-20

[11] Schmidt M 1968 Astrophys. J. 151393

[12] Longair M S 1998 Galaxy Formation (Berlin: Springer) p 392

[13] Guetta D and Piran T 2006 Astron. Astrophys. 453 823-8

[14] Guetta D, Piran T and Waxman E 2005 Astrophys. J. $619412-9$

[15] Virgili F J, Liang E and Zhang B Mon. Not. R. Astron. Soc. 392 91-103

[16] Guetta D and Stella L 2009 Astron. Astrophys. 498 329-33

[17] Dai X 2009 Astrophys. J. 697 L68-71

[18] Salvaterra R and Chincarini G 2007 Astrophys. J. 656 L49-52

[19] Sutton P J, Chatterji S, Jones G, Poprocki S, Rollins J, Searle A, Stein L, Tinto M and Was M 2008 https://geco.phys.columbia.edu/xpipeline/browser/trunk/docs/T080088/T080088-02.pdf

[20] Shibata M and Taniguchi K 2006 Phys. Rev. D 73064027

[21] Shibata M and Uryu K 2002 Prog. Theor. Phys. 107265

[22] Oechslin R and Janka H-T 2007 Phys. Rev. Lett. 99121102

[23] Loffler L, Rezzolla L and Ansorg M 2006 Phys. Rev. D 74104018

[24] Shibata M and Uryu K 2006 Phys. Rev. D 74121503

[25] Faber J A 2006 Astrophys. J. 641 L93

[26] Fryer C L, Holz D E and Hughes S A 2002 Astrophys. J. 565430

[27] van Putten M H et al 2004 Phys. Rev. D 69044007

[28] Smith J R et al 2009 Class. Quantum Grav. 26114013 